\title{
Retrograde Transport of Plasticity Signals in Aplysia Sensory Neurons Following Axonal Injury
}

\author{
John D. Gunstream, Gilbert A. Castro, and Edgar T. Walters \\ Department of Physiology and Cell Biology, University of Texas-Houston Medical School, Houston, Texas 77225
}

Following injury to their peripheral branches, mechanosensory neurons in Aplysia display long-term plasticity that is expressed as soma hyperexcitability, synaptic facilitation, and neurite outgrowth. To investigate the nature of signals that convey information about distant axonal injury, we have investigated the development of injury-induced soma hyperexcitability in two in vitro preparations. In isolated ganglia, proximal nerve crush caused hyperexcitability to appear sooner than did distal crush, and the difference in development of hyperexcitability indicated that the injury signal moved at a rate $(36 \mathrm{~mm} / \mathrm{d})$ similar to previously reported rates of retrograde axonal transport in this animal. This hyperexcitability was not due to interruption of continuous retrograde transport of trophic substances (a negative signal) because inhibitors of axonal transport applied to uncrushed nerve segments did not induce hyperexcitability. Indeed, inhibitors of axonal transport blocked crush-induced hyperexcitability, indicating that positive injury signals are involved. Crush-induced hyperexcitability was unaffected by bathing the nerve in tetrodotoxin or the ganglion in $\mathrm{Cd}^{2+}$, suggesting that the retrograde signals depend upon neither spike activity in the nerve nor synaptic transmission in the ganglion. Close excision of sensory neuron somata (which largely eliminated delays attributable to axonal transport) produced soma hyperexcitability that was expressed after $10 \mathrm{hr}$ and lasted at least $17 \mathrm{~d}$. These data indicate that axonal injury mobilizes signal molecules that are conveyed by retrograde axonal transport into the soma and possibly the nucleus, where they induce long-term plasticity similar to that expressed by these cells during learning and memory.

[Key words: axonal transport, axotomy, hyperexcitability, sensitization, neuronal injury, memory]

Axotomy in most animals is followed by profound reactions in the injured neuron. These include regenerative and degenerative changes in the axon, morphological, and biochemical alterations in the soma ("chromatolysis"), and changes in electrophysiological properties of many parts of the neuron (reviewed by Lieberman, 1971; Fawcett and Keynes, 1990; Titmus and Faber,

\footnotetext{
Received May 2, 1994; revised June 15, 1994; accepted June 23, 1994.

We thank A. Clatworthy, M. Dulin, and P. Illich for comments on an earlier version of this article, J. Pastore for preparing the figures, and N. Karin for use of cell culture facilities. This work was supported by National Science Foundation Grant IBN9210268 to E.T.W. and Program Project Grant P01-DK-37260, NIDDKD, NIH to G.A.C.

Correspondence should be addressed to Edgar T. Walters, Ph.D., Department of Physiology and Cell Biology, University of Texas-Houston Medical School, P.O. Box 20708, Houston, TX 77225.

Copyright (C) 1995 Society for Neuroscience $0270-6474 / 95 / 150439-10 \$ 05.00 / 0$
}

1990). Because many of these alterations involve changes in gene expression and protein synthesis (e.g., Watson, 1968; Herdegen et al., 1992; Haas et al., 1993), pathways must exist that inform the nucleus of cellular damage occurring far from the soma. Many potential injury signals exist, which may act singly or in concert (e.g., Cragg, 1970; Lieberman, 1971; Aldskogius et al.,1992). However, it is useful to distinguish two classes of axonal signals (e.g., Wu et al., 1993). Negative injury signals result from an interruption of retrograde transport of chemical signals, such as trophic substances, that normally are continuously conveyed from distal parts of an uninjured axon to the soma. Positive injury signals, normally absent in uninjured axons, result from the uptake or activation of chemical signals that then move retrogradely from a site of axonal injury. Although most research has focused on negative injury signals (e.g., Fawcett and Keynes, 1990; Titmus and Faber, 1990; Wu et al., 1993), evidence also exists for positive signals (Singer et al., 1982; Soiefer et al., 1988; Ambron et al., 1994). The relative contributions of negative and positive signals to neuronal injury reactions have not been examined systematically in any preparation.

Some reactions to axotomy (notably, hyperexcitability and synaptic facilitation) resemble plastic changes that have been linked to learning and memory (Walters et al., 1991). Neuronal plasticity is defined as a capacity for long-lasting functional modification in response to appropriate stimulation (Konorski, 1948). Molluscan preparations have provided particularly uscful systems for investigating the functions and mechanisms of neuronal plasticity (reviewed by Bulloch, 1985; Carew and Sahley, 1986; Byrne, 1987; Hawkins et al., 1993; Walters, 1994). Nociceptive sensory neurons in the opisthobranch mollusc, Aplysia californica, display long-lasting increases in central and peripheral excitability, in receptive field size, and in synaptic transmission following noxious stimulation of their receptive fields (Walters, 1987a,b; Billy and Walters, 1989). An unexpected finding was that the same plastic changes are produced in Aplysia sensory neurons by crushing axons of the tested cells under conditions that minimize synaptic transmission and spike activity (Walters et al., 1991; Clatworthy and Walters, 1994). This finding, combined with the long latency (1-2 d) of the plastic changes, suggested that some plasticity signals may travel from a crush site to the soma by retrograde axonal transport.

To examine more directly properties of axonal injury signals, we have developed two in vitro preparations that provide experimental control over many factors that may be important in neuronal reactions to injury. We have shown in these preparations that a positive injury signal, carried to the soma by retrograde axonal transport, is required for the induction of electrophysiological changes in the soma. Under our experi- 

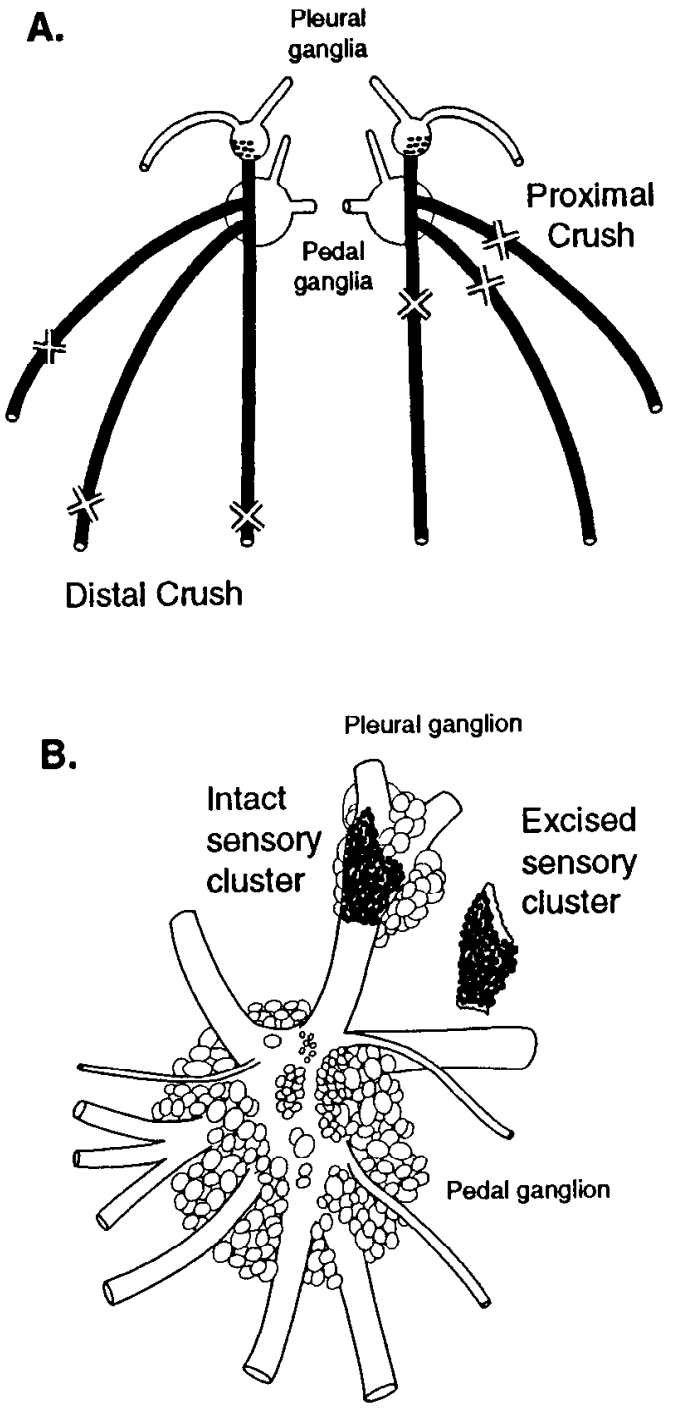

Figure 1. In vitro preparations used to examine electrophysiological alterations of pleural VC sensory neuron somata following axonal injury. $A$, Isolated pleural-pedal ganglia preparation. Dots indicate location of the sensory neuron somata ( 200 in each cluster). Heavy lines indicate sensory neuron axons. Only a few of the pedal nerves are shown. In most experiments, nerves p6, p7, p8, and p9 were crushed. Proximal crush was $10-20 \mathrm{~mm}$ from the sensory neuron somata whereas distal crush in the contralateral nerves was at a distance of $40-60 \mathrm{~mm} . B$, Excised sensory cluster preparation. The cluster was pinned to the substrate after excision, and the remainder of the nervous system discarded.

mental conditions there was no requirement for negative injury signals that might be generated by interrupting retrograde transport of trophic substances. In addition, neither injury-induced action potential discharge nor synaptic release of neuromodulators within the CNS was necessary for inducing plastic changes after axonal injury.

\section{Materials and Methods}

Aplysia californica (100-250 gm) were supplied by Alacrity Marine Biological Services (Redondo Beach, CA) and Aplysia Mariculture Facility (University of Miami). Animals were housed individually in aquaria containing artificial sea water ( $\Lambda$ SW; Instant Ocean, Burlington, $N C)$ at $15-18^{\circ} \mathrm{C}$ for $1-7 \mathrm{~d}$ before use. Constant body weight was maintained on a diet of romaine lettuce.

Prior to each experiment, approximately $20 \mathrm{ml}$ of hemolymph were removed by syringe while the animal was anesthetized by cooling to $0^{\circ} \mathrm{C}$. Additional anesthesia was then provided by injecting sterile, buffered isotonic $\mathrm{MgCl}_{2}$ solution ( $50 \%$ of body weight) at $4^{\circ} \mathrm{C}$. A long incision was made on the dorsal surface of the neck. Pins inserted at the ends of the incision were used to raise the opening above the bath to prevent contamination of the hemolymph. Using sterile tools, each pair of pleural-pedal ganglia was dissected from the animal separately (Fig. 1 $A$ ). All pedal nerves were left as long as possible. Each pair of ganglia was pinned to the Sylgard (Dow Corning, Midland, MI) base of a small Petri dish, and the pleural ganglia desheathed in a filtered and buffered mixture containing equal parts of isotonic $\mathrm{MgCl}_{2}$ solution and ASW. In some cases, pedal nerves were pulled into separate compartments sealed with silicone grease. Absence of leakage was indicated by failure of the phenol red dye in the L- 15 medium to pass from the main compartment into the nerve compartments or, in some experiments, of fast green dye in the nerve compartments to pass into the main compartment. At the end of each experiment nerves were stretched, pinned out, and the distances from the pleural sensory cluster to the crush sites measured. In some experiments, pleural sensory clusters were excised from the desheathed ganglion by undercutting the somata with iridectomy scissors. The remainder of the ganglia were discarded. Two pins inserted through opposite ends of the excised cluster secured it to the Sylgard substrate.

Sterile forceps $(0.1 \mathrm{~mm}$ tip width) were used to crush pedal nerves p6, p7, p8, and p9 (Kandel, 1979) 10-60 mm from the pleural sensory cluster. Nerve crushes were performed in a 1:1 mixture of isotonic $\mathrm{MgCl}_{2}$ and ASW to reduce chemical synaptic transmission and spike activity. Isolated ganglia and excised sensory clusters were bathed in culture medium similar to that described by Schacher and Proshansky (1983). L-15 medium (Sigma, St. Louis, MO) was mixed with the appropriate salts to the following final concentrations: $400 \mathrm{~mm} \mathrm{NaCl} ; 11 \mathrm{mM} \mathrm{CaCl}$; $10 \mathrm{~mm} \mathrm{kCl} ; 27 \mathrm{~mm} \mathrm{MgCl} ; 27 \mathrm{~mm} \mathrm{MgSO}_{4} ; 2 \mathrm{~mm} \mathrm{NaHCO}$. Dextrose $(6 \mathrm{mg} / \mathrm{ml})$, penicillin $\mathrm{G}(25 \mathrm{U} / \mathrm{ml})$, and streptomycin $(25 \mu \mathrm{g} / \mathrm{ml})$ were added. This solution was then mixed 1:1 with sterile filtered Aplysia hemolymph. The cultures were maintained in the dark at $15^{\circ} \mathrm{C}$, and the medium changed every day. In some experiments, the compartment containing pedal nerves was bathed with $60 \mu \mathrm{M}$ tetrodotoxin (TTX, CalBiochem, La Jolla CA) in ASW, $50 \mu \mathrm{M}$ nocodazole (Sigma) in culture medium, or $5 \mathrm{~mm}$ or $20 \mathrm{~mm}$ colchicine (Sigma) in culture medium. Nocodazole was dissolved in dimethyl sulfoxide (DMSO, $5 \mathrm{mg} / \mathrm{ml}$, Sigma) and culture medium.

The effectiveness of nocodazole and colchicine in blocking retrograde axonal transport was tested by axonal backfilling with nickel-lysine dye, using methods described by Fredman (1987). Briefly, the nerves were passed through several chambers: an inner chamber contained both pleural-pedal ganglia bathed in culture medium; two contiguous chambers containing ASW or ASW plus either nocodazole or colchicine; and two outer chambers containing the severed ends of the pedal nerves, which were initially bathed in distilled water to swell the axons and facilitate uptake of dye. After $1 \mathrm{~min}$ in distilled water, an equal volume of nickel-lysine (1 $\mathrm{M} \mathrm{NiCl}_{2}$; Fisher, Fair Lawn, $\mathrm{NJ}$; and $2 \mathrm{M}$ lysine; Sigma) was added. The neurons were allowed to fill for $3 \mathrm{~d}$ at $15^{\circ} \mathrm{C}$. $\mathrm{Ni}^{2+}$ was precipitated by addition of dithiooximide $(5 \mathrm{mg} / \mathrm{ml}$ in DMSO). The ganglia were fixed in glutaraldehyde ( $2 \%$ in phosphate buffer; Sigma), dehydrated in an ethanol series, cleared in methyl salicylate $(1.18 \mathrm{~g} / \mathrm{ml}$; Sigma), and whole mounted on slides for viewing.

Intracellular recordings from sensory neuron somata were made using glass microelectrodes filled with $3 \mathrm{M}$ potassium acetate (electrode resistance 8-20 M 8 ). Recordings were made in $1-2 \mathrm{hr}$ sessions at $17-19^{\circ} \mathrm{C}$ while the ganglia were bathed in buffered ASW (pH 7.6). Between recording sessions the ASW was replaced with culture medium. Sensory neurons were sampled alternately in the left and right clusters, pairing each neuron with a cell in the corresponding location in the contralateral cluster. Up to 16 pairs were sampled per test session. Sampling was restricted to the medial and central parts of the VC cluster, where sensory neurons sending axons out nerves $\mathrm{p} 6, \mathrm{p} 7, \mathrm{p} 8$, and $\mathrm{p} 9$ are concentrated (Billy and Walters, 1987; Dulin et al., 1994). Brief ( $2 \mathrm{msec})$ intracellular depolarizing pulses were used to measure sensory spike amplitude, duration, and afterhyperpolarization. Measurements were only made on spikes that reached threshold after the offset of the depolarizing stimulus. Spike duration was measured from the peak of the spike to the first intersection of the voltage trace with the initial resting potential. Spike threshold in all cells was measured with a standard series of $20 \mathrm{msec}$ depolarizing pulses injected at $1-2 \mathrm{sec}$ intervals until a spike was generated. Beginning at about $0.10 \mathrm{nA}$, currents were initially incremented in $0.05 \mathrm{nA}$ steps, then in $0.02 \mathrm{nA}$ steps, and finally in 0.01 steps when 
the resulting depolarizations exceeded approximately $20 \mathrm{mV}$. Excitability was measured by counting the number of spikes fired in response to a $1 \mathrm{sec}$ intracellular depolarizing pulse using $1.25 \times$ and $2.5 \times$ the threshold current determined with the $20 \mathrm{msec}$ pulse. Input resistance was determined by injecting a $1 \mathrm{sec}, 0.5 \mathrm{nA}$, hyperpolarizing pulse (after rebalancing the bridge). In experiments involving unilateral treatment with TTX, nocodazole, or colchicine, all electrophysiological tests were conducted by an experimenter "blind" to the nerve treatment.

Most statistical comparisons were made with paired, two-tailed $t$ tests. A probability $(p)$ of less than 0.05 was considered significant. In some studies, the average values for each cluster (sampling 12-16 cells) were used as data points for paired comparisons across several animals. Thus, in these cases the individual subjects in the analysis were sensory clusters. However, in other studies we took advantage of the large number of cells in each cluster and made the subjects of the analysis individual cells. This permitted rigorous statistical tests of a given hypothesis within a single animal, using two paired populations of sensory neurons from each cluster. To ensure that a statistically significant result obtained within a single animal was repeatable across animals, all such experiments were replicated in at least one additional animal. In one study, an analysis of variance (ANOVA) was performed on independent measurements from different sensory neurons sampled during three separate periods in pleural ganglia from the same animal. Subsequent inspection of differences between pairs of means was conducted with NewmanKeuls tests.

\section{Results}

Sensory neuron excitability differs after proximal and distal nerve crush in vitro

If axonal injury signals depend upon retrograde axonal transport, reactions of the soma to proximal axon crush should occur considerably earlier than reactions to distal axon crush (Fig. $1 A$ ). We first tested this prediction in vitro by examining the soma excitability of sensory neurons 1 to $2 \mathrm{~d}$ after crushing pedal nerves $\mathrm{p} 6, \mathrm{p} 7, \mathrm{p} 8$, and $\mathrm{p} 9$ in six animals. This period was selected because a previous study in the intact animal suggested that the latency for hyperexcitability after relatively distal crush is about $2 \mathrm{~d}$ (Walters et al., 1991). As illustrated in Figure 2A, sensory neurons whose axons were crushed about $20 \mathrm{~mm}$ from their somata were more excitable during this period than contralateral sensory neurons whose axons were crushed $40-60 \mathrm{~mm}$ from their somata. The mean ( \pm SEM) number of spikes evoked by a 1 sec depolarizing pulse (using a current 2.5 times the threshold current for evoking a spike with a $20 \mathrm{msec}$ pulse) was $6.6 \pm 0.5$ and $4.9 \pm 0.4$ spikes after proximal and distal crush, respectively $[t(5)=6.86, p<0.001]$. This increase in excitability of the soma is likely to contribute to the afterdischarge generated in the soma, which amplifies nociceptive input to the CNS during sensitization (Clatworthy and Walters, 1993). As illustrated in Figure $2 B$, nerve crush also caused other plastic changes in the sensory neuron soma, including prolongation of the action potential and reduction of the AHP. These additional plastic changes are analyzed in a subsequent section.

\section{Time course of hyperexcitability after proximal and distal nerve crush}

Consistent differences in the development of sensory neuron soma hyperexcitability after proximal and distal nerve crush provide an estimate of the rate that the inducing signals move from the crush site to the soma. To investigate the development of crush-induced hyperexcitability, we tested separate groups of sensory neurons at various intervals between 6 and $96 \mathrm{hr}$ after nerve crush (Fig. 3). Two to four animals were examined at each point, and each animal was examined only once. The mean distances of the proximal and distal crushes from the ipsilateral
Distal crush

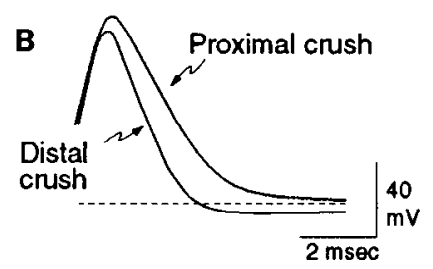

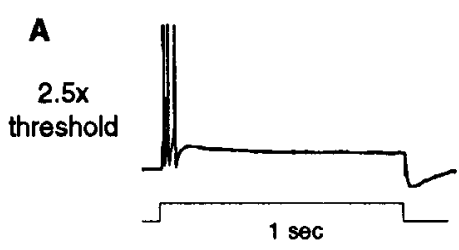

Figure 2. Examples of electrophysiological alterations $30 \mathrm{hr}$ after proximal nerve crush in vitro. $A$, Enhanced soma excitability shown by greater spike number during prolonged depolarization in a sensory neuron given proximal axonal crush compared to a paired cell in the contralateral cluster given distal axonal crush. A 1 sec depolarizing pulse was delivered to the soma through the recording electrode. Stimulus intensity was $2.5 \times$ the current required to reach spike threshold during a 20 msec pulse. Spike amplitudes are attenuated by the limited sampling rate of the data acquisition system. $B$, Increased spike duration and decreased AHP in a sensory neuron given proximal axonal crush relative to the paired contralateral cell given distal axonal crush. Each spike was initiated by a $2 \mathrm{msec}$ depolarizing pulse (not shown) that ended just before spike threshold was reached. The dashed line indicates initial resting potential.

pleural sensory cluster in this phase of the study were $10.4 \mathrm{~mm}$ and $43.8 \mathrm{~mm}$, respectively.

Sensory neurons whose axons received proximal crush began to show an increase in excitability after approximately 18 to 24 $\mathrm{hr}$, while sensory neurons that received distal crush began to show an increase in excitability after 30 to $36 \mathrm{hr}$ (Fig. $3 A$ ). In each case, excitability continued to increase before peaking at the same level of 9-10 spikes/ 1 sec pulse $72-96 \mathrm{hr}$ after crush. The mean differences in excitability between sensory clusters within individual animals are shown as data points in Figure 3B. Statistically significant hyperexcitability (for proximal vs. distal crush) was observed in one of two animals tested $18 \mathrm{hr}$ after crush, in all animals tested 24, 30, and $36 \mathrm{hr}$ after crush, and in one of two animals tested $48 \mathrm{hr}$ after crush. As previously observed in sensory neurons with uncrushed axons (Walters et al., 1983a), none of the sensory neurons in these experiments ( $n=20$ animals and 560 cells) displayed any action potentials in the absence of test stimulation (see below).

The rate that the inducing signals move from the crush site to the soma was estimated by calculating the average temporal offset between points of identical excitability on the ascending limbs of the curves shown in Figure $3 A$. Linear regression was performed on data collected between 24 and $36 \mathrm{hr}$ in the proximal crush group and between 30 and $48 \mathrm{hr}$ in the distal crush group. The temporal offsets between the lines at excitability levels of four spikes and six spikes were averaged. The mean temporal offset of $21.3 \mathrm{hr}$ and the mean difference in distance of $32 \mathrm{~mm}$ yielded an estimated rate of $36 \mathrm{~mm} / \mathrm{d}$.

\section{Soma hyperexcitability is delayed for $10 \mathrm{hr}$ after axonal plasticity signals reach the soma}

In principle, the delay in expression of soma hyperexcitability following axonal injury can be divided into the time required 
A.

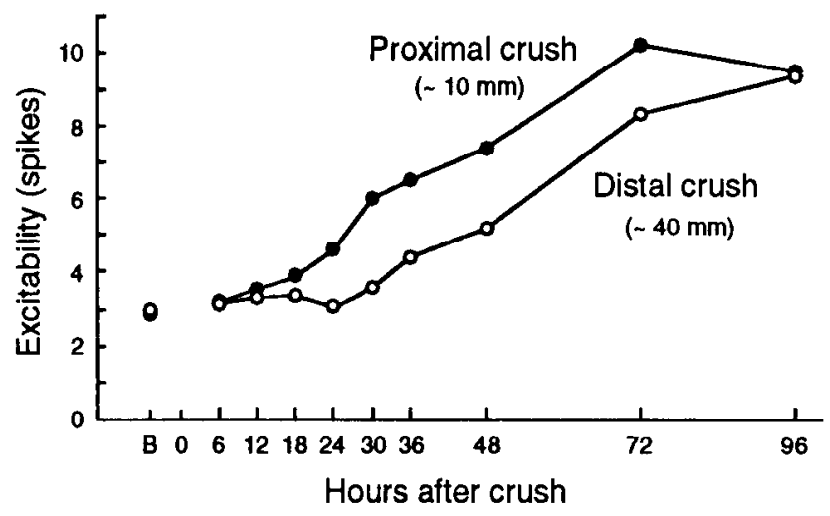

B.

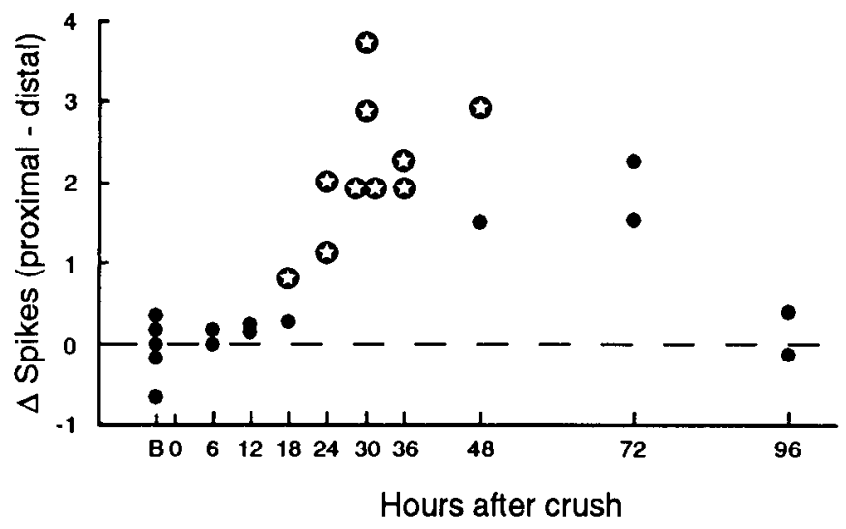

Figure 3. Development of soma hyperexcitability following proximal and distal nerve crush. $A$, Mean number of spikes evoked in the $2.5 \times$ threshold excitability test (see Fig. $2 A$ ). Each point represents data from two to five animals, with 14-16 cells tested per cluster, except for baseline tests $(B)$ where six to eight cells per cluster were tested. In each case, one cluster received proximal crush ( filled circles) and one received distal crush (open circles). B, Mean difference in spikes evoked in each cell pair (proximal crush - distal crush) plotted for each animal contributing to the data shown in part $A$. Stars indicate individual animals in which cells receiving proximal crush were significantly more excitable than paired cells receiving distal crush $(p<0.05)$. Two of the four stars at $30 \mathrm{hr}$ represent identical values, and are offset slightly so that both can be seen on the graph.

for (1) retrograde transport of plasticity signals in the axon and (2) processes occurring in the soma after receipt of the axonal plasticity signals. We used two methods to estimate the contribution of the soma to this delay. First, combining the rate of $36 \mathrm{~mm} / \mathrm{d}$ for retrograde movement of the inducing signals with the distances of the proximal $(10 \mathrm{~mm})$ and distal $(40 \mathrm{~mm})$ crush sites (Fig. 3), we calculated that the axonal injury signals should have arrived in the soma approximately $7 \mathrm{hr}$ after proximal crush and $27 \mathrm{hr}$ after distal crush. This was $11 \mathrm{hr}$ prior to the first observation of significant hyperexcitability following proximal crush, and $9 \mathrm{hr}$ prior to the first observation of significant hyperexcitability following distal crush. Thus, both observations yielded an estimate of approximately $10 \mathrm{hr}$ for the delay attributable to processing of injury signals after they had been received in the soma.

An independent estimate of the contribution of the soma to the delay in expression of plasticity was obtained by measuring the time course of hyperexcitability under conditions in which the delay attributable to axonal transport was negligible. This was accomplished by transecting the sensory neurons within about $100 \mu \mathrm{m}$ of the soma, i.e., by close excision of the cluster of sensory neuron somata (Fig. $1 B$ ). If the rate of retrograde axonal transport is uniform throughout the axon, the estimated rate of $36 \mathrm{~mm} / \mathrm{d}$ would translate into a delay of as little as 4 min for the signals to get to the soma from a transection site $100 \mu \mathrm{m}$ away. We began by making sure that this procedure, like the axonal crush procedure, would produce hypcrexcitability after a sufficient delay. We excised one sensory cluster 18 $\mathrm{hr}$ before testing. The second (contralateral) cluster was surgically desheathed at the time the first cluster was excised, but it was not excised until $2 \mathrm{hr}$ before testing. The cluster that had been excised for $18 \mathrm{hr}$ displayed significantly more spikes in the $2.5 \times$ threshold excitability test than the cluster that had been excised for only $2 \mathrm{hr}[7.3 \pm 1.2$ and $3.3 \pm 0.4$ spikes, respectively; $t(9)=2.78, p<0.02$ ]. Like the sensory neurons subjected to axon crush, none of the excised sensory neurons displayed any spontaneous activity ( $n=4$ animals and 158 cells).

To see when hyperexcitability first became evident in this preparation, we sampled sensory neurons $1 \mathrm{hr}$ before excision and then took additional samples from the same two clusters (from a single animal) at various times afterwards. In initial studies ( $n=3$ animals), we found that hyperexcitability first became apparent $10 \mathrm{hr}$ after excision, and persisted for at least $17 \mathrm{~d}$ (Fig. $4 A$ ). We tested this prediction explicitly in an experiment in which we compared excitability of sensory neurons sampled 10 to $12 \mathrm{hr}$ after excision to excitability sampled in different sensory neurons within the same cluster either 7.5 to $9.5 \mathrm{hr}$ after excision or prior to excision (Fig. $4 B$ ). A one-way ANOVA revealed a significant difference among these groups $[F(2,37)=6.66, p<0.005]$. Post hoc comparisons with the Newman-Keuls test showed that excitability sampled in cells $10-12.5 \mathrm{hr}$ after excision was significantly greater than excitability measured $7.5-9.5 \mathrm{hr}$ after excision $(p<0.005)$ or during baseline testing before excision $(p<0.05)$. Excitability sampled 7.5-9.5 hr after excision was not significantly different from excitability sampled during baseline. These results indicate that, after the soma receives axonal injury signals, there is a delay of about $10 \mathrm{hr}$ for the soma to process these signals and respond with an increase in excitability.

\section{Additional soma properties altered by axon injury in vitro}

How similar are the effects on sensory neurons of nerve injury in vitro to previously described effects in the same cells produced by axonal injury in vivo? We addressed this question by examining the same set of electrophysiological properties after nerve crush in the isolated ganglia that had becn cxamined previously after nerve crush in the intact animal. Table 1 summarizes data collected from animals that were tested 24, 30, and $36 \mathrm{hr}$ after proximal and distal nerve crush. The data are taken from the animals whose soma excitability is plotted in Figure 3. Soma excitability, as judged by the number of spikes evoked by a $1 \mathrm{sec}$ depolarizing pulse, was significantly greater in cells undergoing proximal axonal crush than distal axonal crush. This was true for both a weak depolarizing pulse (1.25 $x$ the $20 \mathrm{msec}$ threshold) and a strong depolarizing pulse (2.5 $x$ threshold). In addition, cells undergoing proximal axonal crush had significantly longer spike durations, smaller spike afterhyperpolarizations (AHP), and lower thresholds. No significant differences were observed in spike amplitude, input resistance, or resting potential. It should be noted that sensory neurons given proximal crush responded with more spikes in 
A.
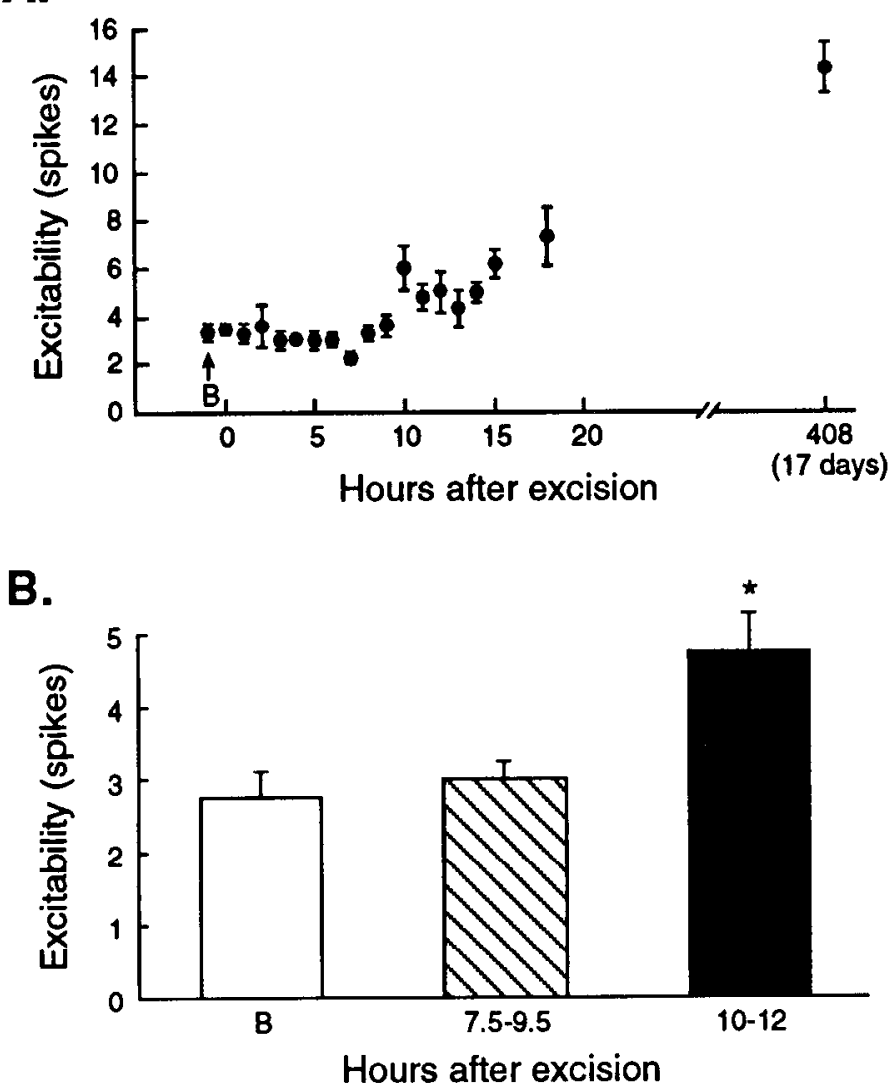

Figure 4. Hyperexcitability induced by close excision of the sensory neuron cluster is expressed after a $10 \mathrm{hr}$ delay. $A$, Pooled data (mean + SFM) from 109 cells sampled at various times before and after excision of six pleural sensory clusters in three animals. Experiments were run at $18-19^{\circ} \mathrm{C}$. In one animal, an additional 10 cells were sampled 17 $\mathrm{d}$ after excision. $B$, Test of hypothesis that latency of hyperexcitability after soma excision is $10 \mathrm{hr}$. Independent samples were taken from both sensory clusters in a single animal before excision, 7.5-9.5 hr after excision, and 10-12 hr after excision $(n=8,16$, and 16 cells, respectively). Star indicates significant hyperexcitability relative to the other groups of cells $(p<0.05)$

the excitability tests, even though less current was usually injected into their somata than into the somata of sensory neurons given distal crush (because the injected current was normalized to spike threshold, which was usually lower after proximal crush). Thus, differences in injected current cannot account for the observed differences in excitability.

Similar effects were observed following excision of the sensory neuron somata. In the experiment shown in Figure $4 B$, cells examined 10-12.5 hr after excision were compared to cells examined 7.5-9.5 $\mathrm{hr}$ after excision. At the later time point, the sensory neurons displayed significantly greater excitability [4.8 vs. 3.0 spikes in the $2.5 \times$ threshold test, $t(15)=2.70, p<$ $0.05]$, longer spike durations [4.7 vs. $3.8 \mathrm{msec}, t(15)=2.55, p$ $<0.05]$, and smaller afterhyperpolarizations $[2.1$ vs. $3.3 \mathrm{mV}$, $t(15)=2.66, p<0.05]$.

In contrast to the significant differences in excitability that were frequently seen within a single animal (Fig. $3 B$ ), changes in the other electrophysiological properties were often only statistically significant when comparisons of averaged responses were made across several animals (Table 1). The robustness of the alterations in excitability may reflect, at least in part, the
Table 1. Electrophysiological properties altered 24-36 hr after proximal nerve crush in vitro

\begin{tabular}{lccl} 
Property & Distal crush & Proximal crush & $p$ \\
\hline Excitability (spikes) & & & \\
$\quad$ & & & \\
$\quad 2.5 \times$ threshold & $1.3 \pm 0.1$ & $2.0 \pm 0.3$ & 0.01 \\
Threshold (nA) & $3.4 \pm 0.3$ & $5.9 \pm 0.5$ & 0.001 \\
Spike duration (msec) & $0.83 \pm 0.08$ & $0.70 \pm 0.07$ & 0.01 \\
AHP (mV) & $3.8 \pm 0.2$ & $4.2 \pm 0.3$ & 0.05 \\
Spike amplitude (mV) & $77.5 \pm 0.4$ & $5.0 \pm 0.4$ & 0.02 \\
Input resistance $(\mathrm{M} \Omega)$ & $33.6 \pm 5.5$ & $77.6 \pm 1.1$ & 0.76 \\
Resting potential $(\mathrm{mV})$ & $44.0 \pm 0.7$ & $44.8 \pm 0.9$ & 0.24
\end{tabular}

Data are from eight animals (116 cell pairs) and are expressed as means \pm SEM Values of $p$ were determined with two-tailed paired $t$ tests with $n=8$. AHP, afterhyperpolarization. Excitability refers to the number of spikes elicited by a $1 \mathrm{sec}$ depolarizing pulse at $1.25 \times$ or $2.5 \times$ the $20 \mathrm{msec}$ spike threshold. Data were taken from the experiments plotted in Figure 3.

absolute precision with which spike number can be determined. Determinations of spike threshold, duration, AHP, and input resistance have larger intrinsic errors, especially when a single intracellular electrode is used for both current injection and voltage measurement.

\section{Injury-induced hyperexcitability does not require spike activity or synaptic transmission}

In principle, delayed effects of nerve crush might be produced by immediate or delayed injury discharge in the damaged axons. These action potentials could act as a direct signal to the soma, or act indirectly via synaptic excitation of interncurons that modulate the excitability of the sensory neuron soma. Mammalian sensory neurons often show prolonged spontaneous activity following nerve injury (e.g., Wall and Gutnick, 1974; Kocher et al., 1987; Kajander and Bennett, 1992), and a gradual buildup of the effects of such activity might constitute a slow injury signal. Although injury discharge and its effects were minimized during nerve crush in the experiments described thus far by bathing the preparations in a solution containing elevated $\mathrm{Mg}^{2+}$ and reduced $\mathrm{Na}^{+}$concentrations, the possibility remained that sufficient activity was generated to trigger plasticity, or that activity-dependent plasticity developed during some period after the preparation was returned to the culture medium. To eliminate or greatly reduce activity-dependent effects of nerve crush, we bathed the crush site in $60 \mu \mathrm{M}$ TTX during the crush and throughout the remainder of the experiment. In the same experiments, the ganglia were bathed in $2 \mathrm{mM} \mathrm{Cd}^{2+}$ for $10 \mathrm{~min}$ before and $20 \mathrm{~min}$ after the nerve crush. Intracellular recordings (not shown) confirmed that the TTX blocked all spike activity and that the $\mathrm{Cd}^{2+}$ blocked all postsynaptic potentials recorded in pleural ganglion sensory and interneurons during intense nerve stimulation. Figure 5 shows that the combination of peripheral TTX and central $\mathrm{Cd}^{2+}$ did not prevent soma hyperexcitability $30 \mathrm{hr}$ after proximal nerve crush. In both animals examined under these conditions, cells whose axons received proximal crush showed significant hyperexcitability compared to paired contralateral cells whose axons received distal crush $[t(12)=$ $3.75, p<0.01$ and $t(14)=4.67, p<0.01$ ]. Similar results (not shown) were obtained when $\mathrm{Cd}^{2+}$ was not used and TTX was applied for $30 \mathrm{hr}$ to the nerves receiving the proximal crush while the nerves receiving distal crush were bathed in ASW $[t(12)=4.03$ and $t(14)=3.88, p<0.01$ in each case]. Bathing 


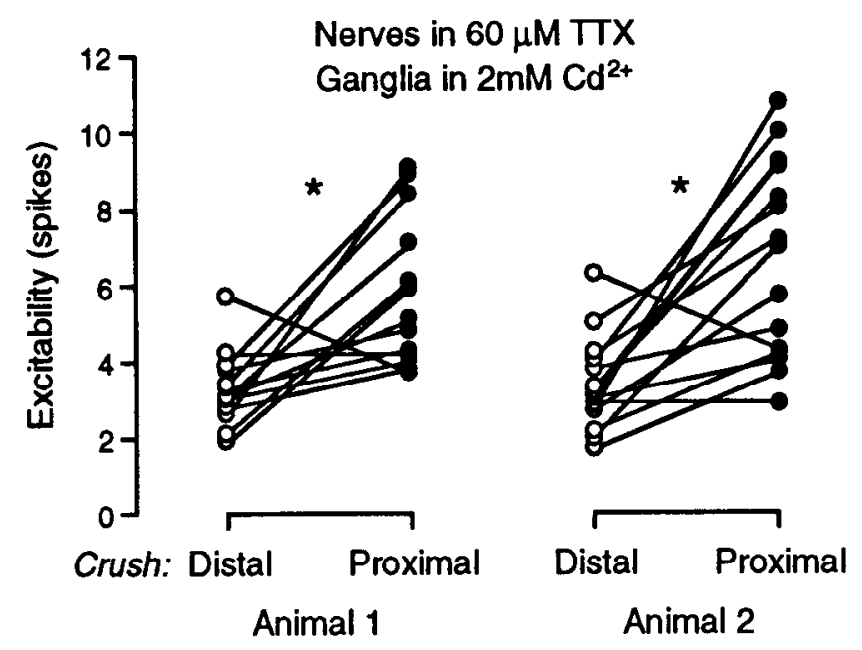

Figure 5. Hyperexcitability is not prevented by blocking peripheral spike activity and central synaptic transmission. The nerves were bathed in $60 \mu \mathrm{M}$ TTX continuously from $10 \mathrm{~min}$ before to $30 \mathrm{hr}$ after the crush. The ganglion was bathed in $2 \mathrm{mM} \mathrm{Cd}^{2+}$ from $10 \mathrm{~min}$ before to $20 \mathrm{~min}$ after the nerve crush. Lines connect pairs of sensory neurons sampled from corresponding locations within the VC cluster. Hyperexcitability after proximal crush is indicated by a positive slope. Many of the cells examined in the control condition (distal crush) showed equal excitability. In these cases, both the control and proximal crush values in each of the affected pairs are offset slightly to allow visualization of individual points. These small shifts are balanced around the true value, and do not change the slopes of the lines. The mean values for the proximal and distal crushes were, respectively: animal $1-3.3$ and 5.8 spikes $(n=13$ pairs); animal $2-3.3$ and $6.6(n=15$ pairs). Stars indicate $p<0.01$ for differences between distal and proximal crush.

distally crushed nerves in TTX for $30 \mathrm{hr}$ while the contralateral nerves were bathed in ASW revealed no apparent effects of TTX application on soma excitability ( $n=9$ cell pairs). In two other animals (not shown) we compared the $30 \mathrm{hr}$ excitability of sensory neurons undergoing proximal crush to the excitability of contralateral sensory neurons undergoing proximal crush in the presence of TTX. The lack of any significant difference between the two sides ( $n=24$ cell pairs) indicates that injury-induced hyperexcitability in the presence of TTX is indistinguishable from that observed in the absence of TTX under these conditions.

\section{Interruption of axonal transport does not induce hyperexcitability}

A second potential signal for delayed hyperexcitability is an injury-induced interruption of continuous retrograde axonal transport of trophic signals from the periphery. We began to test this possibility using ganglia that had been isolated with nerves $\mathrm{p} 8$ and $\mathrm{p} 9$ left as long as possible $(4-6 \mathrm{~cm})$. As shown in Figure 3, crush at this distance causes little if any change in soma excitability after $30 \mathrm{hr}$. Microtubule depolymerizers known to disrupt axonal transport in Aplysia (Koike et al., 1989; Ambron et al., 1992) were applied to proximal parts of the nerves. Nerves from the contralateral pedal ganglia were treated identically except that culture medium alone, rather than a microtubule depolymerizer in culture medium, was put into the well containing the proximal part of the nerves. Blockade of retrograde axonal transport was confirmed by demonstrating that each microtubule depolymcrizer blocked the rapid movement of nickel-lysine dye into the ganglia in axonal backfilling experiments (not shown). During the same $72 \mathrm{hr}$ period contralateral nerves in ASW showed clear retrograde transport of the nickel-lysine into the ganglia. No significant hyperexcitability was produced by $30 \mathrm{hr}$ application to the nerve of $5 \mathrm{~mm}$ or 20 $\mathrm{mM}$ colchicine or $50 \mu \mathrm{M}$ nocodazole at a distance of $1-2 \mathrm{~cm}$ from the pleural cluster (Fig. 6). Because DMSO was used to help dissolve the nocodazole in culture medium, we tested whether DMSO might produce a depression of soma excitability that would obscure a potential enhancement of excitability by nocodazole. Application of DMSO alone for $30 \mathrm{hr}$ had no significant effect on excitability (not shown). Taken together, these data indicate that interruption of retrograde axonal transport is

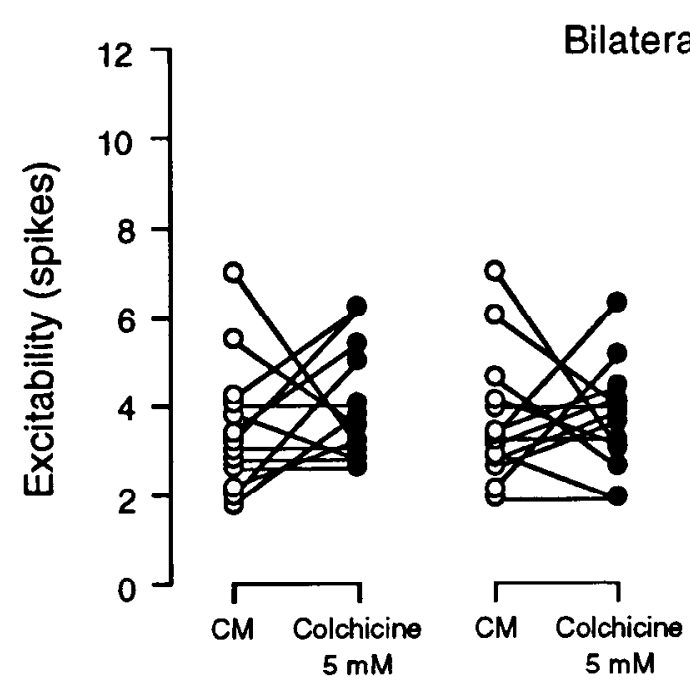

Animal 1

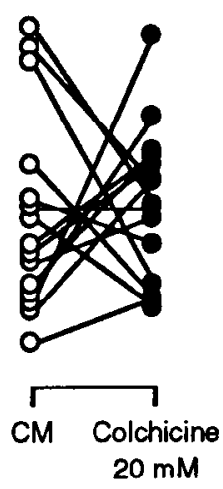

Animal 3

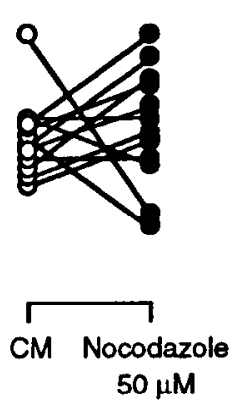

Animal 4
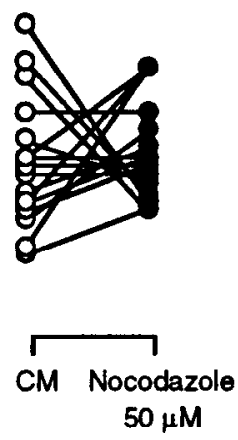

Animal 5

Figure 6. Interruption of axonal transport in undamaged nerve segments does not induce hyperexcitability of the sensory neuron soma. The mean values for somata with axons in nerves bathed in culture medium $(C M)$ or the indicated microtubule depolymerizer in CM (and DMSO for nocodazole) were, respectively: animal $1-3.9$ and 3.5 spikes $(n=13$ pairs); animal $2-3.6$ and $3.5(n=15$ pairs); animal $3-4.1$ and $3.9(n=15$ pairs); animal $4-3.9$ and $3.8(n=12$ pairs); animal $5-4.3$ and $3.9(n=16$ pairs). For all differences $p>0.30$. 


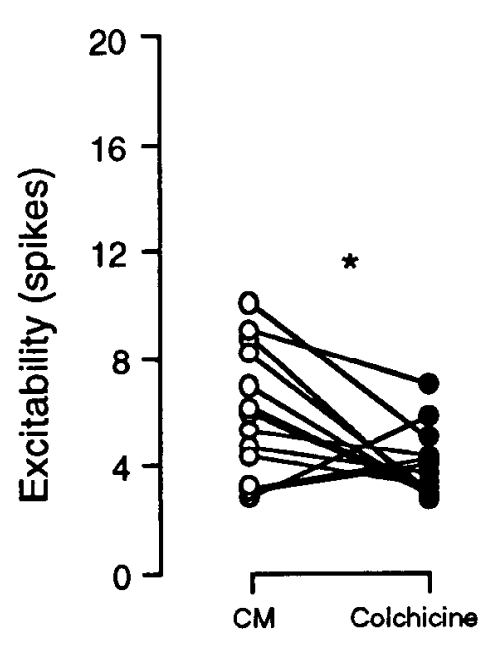

Animal 1

\section{Bilateral proximal crush}

Animal 2

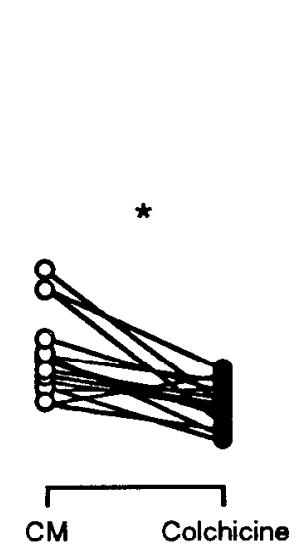

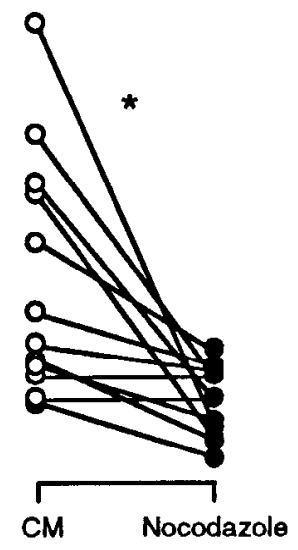

Animal 3

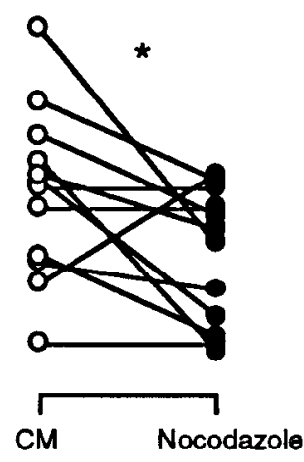

Animal 4

Figure 7. Interruption of axonal transport blocks hyperexcitability observed $30 \mathrm{hr}$ after proximal nerve crush. Lack of hyperexcitability after proximal crush in the presence of the indicated microtubule depolymerizer is indicated by a negative slope. The mean values for somata with axons in nerves bathed in culture medium $(C M)$ or either $5 \mathrm{mM}$ colchicine in CM or $50 \mu \mathrm{M}$ nocodazole in CM and DMSO were, respectively: animal $1-6.1$ and $3.9(n=14$ pairs); animal $2-4.9$ and $3.0(n=15$ pairs); animal $3-7.5$ and $3.0(n=12$ pairs); animal $4-7.6$ and $5.3(n=12$ pairs) Stars indicate $p<0.05$ for differences between nerve treatment with ASW and treatment with the microtubule depolymerizer.

not responsible for the hyperexcitability observed $30 \mathrm{hr}$ after proximal nerve crush.

\section{Interruption of axonal transport blocks crush-induced hyperexcitability}

Another hypothesis to explain the results shown in Figure 3 is that soma hyperexcitability following axon injury depends upon positive molecular signals generated at the site of axonal injury and slowly conveyed by retrograde axonal transport to the soma. A prediction of this hypothesis is that interrupting retrograde transport with microtubule depolymerizers should prevent the injury-induced hyperexcitability. We tested this prediction with bilateral proximal crushes, where one side was crushed in culture medium alone and the other in the presence of colchicine or nocodazole. As shown in Figure 7, significant attenuation of the hyperexcitability induced $30 \mathrm{hr}$ after proximal nerve crush was produced by application to the nerve of both $5 \mathrm{~mm}$ colchicine $[t(13)=3.08$ and $t(14)=4.40, p<0.01$ in each case $]$ and 50 $\mu \mathrm{M}$ nocodazole $[t(12)=3.33$ and $2.44, p<0.05$ in each case $]$.

\section{Discussion}

The present study has shown that sensory neuron plasticity similar to that found after nerve crush in intact anesthetized animals (Walters et al., 1991; Clatworthy and Walters, 1994) occurs after nerve crush in the isolated nervous system and after excision of sensory neuron somata. These reduced preparations have provided direct support for the hypothesis that positive injury signals are conveyed to the neuronal soma by retrogradc axonal transport.

\section{Evidence for retrograde axonal transport of positive injury signals}

Three findings strongly indicate that injury signals are conveyed to the sensory neuron soma by retrograde axonal transport. First, we found in an in vitro preparation, as previously shown in vivo (Walters et al., 1991; Clatworthy and Walters, 1994), that the latency for expression of hyperexcitability following nerve injury is $1-2 \mathrm{~d}$, depending upon the distance to the site of axonal injury from the sensory neuron somata. In the present study we were able to estimate a transport rate $(36 \mathrm{~mm} / \mathrm{d})$ that is consistent with the minimal rate of $25 \mathrm{~mm} / \mathrm{d}$ for retrograde axonal transport determined by Ambron et al. (1992) for Aplysia neurons in culture. Second, we found that injury-induced hyperexcitability was not due to slowly developing consequences of activity-dependent signals, since it was unaffected by blocking TTX-sensitive spike activity in injured nerves or by blocking synaptic transmission within the CNS. This conclusion was further supported by the complete absence of spontaneous action potentials observed in recordings from 718 sensory neurons made $1 \mathrm{hr}$ to $17 \mathrm{~d}$ after axonal injury (see Figs. 3 and 7 ). Third, injury-induced hyperexcitability was prevented by application to the crush site of microtubule depolymerizers that we showed would disrupt axonal transport in these cells (see also Koike et al., 1989; Ambron et al., 1992).

The blockade by colchicine and nocodazole of hyperexcitability $30 \mathrm{hr}$ after proximal crush indicates that the hyperexcitability is triggered by a positive axonal injury signal. If hyperexcitability were triggered by a negative signal, due to interruption of tonic retrograde signals such as trophic substances, disruption of axonal transport in undamaged lengths of nerve would be expected to induce rather than block this plasticity. However, $30 \mathrm{hr}$ application of colchicine or nocodazole to long uncrushed nerves had no effect on soma excitability. Additional support for a positive molecular injury signal has recently come from studies of axoplasmic proteins in Aplysia. Some of these proteins contain nuclear localization sequences (Schmied et al., 1993) that cause them to be transported to the soma and imported into the nucleus. Retrograde transport into the soma of at least one of these proteins is triggered by axonal injury (Ambron et al., 1994). Walters et al. (1994) collected material transported retrogradely from a crush site to a ligation and found that injection into the sensory neuron of this material caused effects 
on soma electrophysiology that were remarkably similar to the effects of axon damage described in the present studies.

The results of Ambron et al. (1994) and Walters et al. (1994), combined with evidence for the specificity of crush-induced plastic changes to cells with injured axons (Clatworthy and Walters, 1994) and evidence in the present article that plasticity signals move by retrograde axonal transport, indicate that injury of axons and/or nearby cells causes specific signal molecules to enter the retrograde axonal transport pathway. Once the signal molecules reach the soma there is a $10 \mathrm{hr}$ delay before the soma expresses hyperexcitability. This long delay suggests that the signal molecules do not directly modulate ionic channels controlling excitability. In addition, the $10 \mathrm{hr}$ delay in the soma excision experiments is too long to be due to diffusion of second messengers such as cAMP that might be generated at axonal transection sites a few hundred $\mu \mathrm{m}$ from the soma (see also Bacskai et al., 1993; Kasai and Petersen, 1994). Recently, we found (Gunstream et al., 1994) that inhibitors of protein synthesis and gene transcription block the hyperexcitability that occurs $18 \mathrm{hr}$ after excision of sensory neuron somata. These observations are consistent with the possibility that the signal molecules are proteins that are imported into the nucleus by virtue of their nuclear localization sequences (Schmied et al., 1993). In the nucleus, these molecules may induce plastic changes by regulating gene transcription, perhaps in a manner similar to that which occurs during prolonged application of 5-HT to the soma (Montarolo et al., 1986; Dale et al., 1987) or the periphery (Clark and Kandel, 1993).

Although we found no evidence that negative axonal injury signals are involved in triggering long-term hyperexcitability after axonal injury, one should not conclude that negative signals are unimportant. Given ample evidence that trophic factors from target tissues maintain differentiated neuronal properties in diverse systems (e.g., Titmus and Faber, 1990) and the influence of postsynaptic targets on the growth of neurites in Aplysia sensory neurons (Glanzman et al., 1989), it seems likely that injury-induced interruption of signals coming from targets will have some effect on these cells. Other potential effects (e.g., morphological changes) and additional time points need to be examined to see what influences negative axonal injury signals have in these cells.

\section{What activates the positive axonal injury signals?}

In principle, many potential consequences of nerve injury might cause signal molecules to enter the retrograde transport/nuclear import pathway, including direct effects of axonal injury (e.g., $\mathrm{Ca}^{2+}$ influx or disruption of the cytoskeleton). Although we have not yet identified the earliest triggers of injury-induced plasticity, some potential signals have been excluded by our experiments. The failure to find hyperexcitability in sensory neurons that had been desheathed $18 \mathrm{hr}$ earlier but only excised $2 \mathrm{hr}$ earlier (in contrast to the hyperexcitability in contralateral cells that had been desheathed and excised $18 \mathrm{hr}$ earlier) suggests that the presence of sheath near the axonal injury site is not required to generate plasticity signals, and that cutting nearby sheath is not sufficient to generate these signals. This result is consistent with the possibility that positive injury signals are generated initially in damaged sensory neuron axons and/or within cells in very close proximity to the damaged axons.

If factors released from surrounding cells are critical for inducing soma hyperexcitability, the release of such factors during injury is unlikely to require normal synaptic transmission, be- cause the high $\mathrm{Mg}^{2+}$ concentrations in our experiments $(3.5 \times$ normal) greatly reduces chemical synaptic transmission in $A p l y$ sia. Both the induction of hyperexcitability in the presence of central $\mathrm{Cd}^{2+}$ and its expression during prolonged bathing of the crushed nerve in TTX indicate that the hyperexcitability is not secondary to injury-induced activation of modulatory neurons that release facilitatory neurotransmitters onto sensory neurons (e.g., Mackey et al., 1989). This inference is further supportcd by the observation that sensory neurons with uncrushed axons following nearby nerve crush do not exhibit hyperexcitability (Clatworthy and Walters, 1994). It is also consistent with the absence of behavioral sensitization in the intact animal following nerve crush under strong anesthesia (Walters et al., 1991).

Another set of extracellular factors that might lead to intraaxonal injury signals may come from amebocytes attracted to a wound (e.g., Lu and Richardson, 1991; Clatworthy et al., 1994). Because blood-borne amebocytes in our in vitro preparation were eliminated by filtering the culture medium, the only available amebocytes would have to have been already present within nervous tissue. Host defense processes can produce hyperexcitability in these sensory neurons when activated by a foreign body near the nerve in the intact animal, but this effect occurs with a latency of about $5 \mathrm{~d}$ (Clatworthy et al., 1994). The development of hyperexcitability within $10 \mathrm{hr}$ of soma excision suggests that this plasticity can occur before amebocytes have time to aggregate at a site of injury. However, under natural conditions, host defense cells may be involved in inducing or maintaining neuronal hyperexcitability after injury. Indeed, the greater degree of nerve crush-induced hyperexcitability in vivo than in vitro might reflect synergism of intrinsic axonal injury signals with factors (c.g., cytokines) released by host defense cells in vivo.

\section{Injury reactions and memory mechanisms}

Similar changes in excitability, synaptic transmission, and morphology in Aplysia sensory neurons are observed after axonal injury (Walters et al. 1991; Dulin and Walters, 1993; Clatworthy and Walters, 1994; Dulin et al., 1994) and learning (e.g., Hawkins et al., 1983; Walters et al., 1983b; Klein et al., 1986; Scholz and Byrne, 1987; Walters, 1987b; Bailey and Chen, 1988; Billy and Walters, 1989; Nazif et al., 1991). This similarity suggests that injury-induced alterations are likely to be present in studies of long-term, learning-related plasticity induced by $5-\mathrm{HT}$ or cAMP, both in isolated ganglia (e.g., Eskin et al., 1989; Nazif et al., 1991; Noel et al., 1991) and dissociated cells in culture (e.g., Montarolo et al., 1986; Dale et al., 1987; Dash et al., 1990; Mayford et al., 1992). Indeed, Alberini ct al. (1994) have shown that dissection activates the same immediate-early gene, C/EBP, that they showed to be necessary for 5-HT-induced long-term synaptic facilitation in culture. Although injury-induced background alterations have not prevented the recognition of important differential effects of experimental and control treatments, some molecular mechanisms of memory might be missed if the same mechanisms are induced in control cells as a consequence of dissection. Conversely, the possibility that learning mechanisms and adaptive injury mechanisms overlap suggests that studies of injury reactions may provide insights into primitive memory mechanisms. For example, a hypothesis based on molecular reactions to axonal injury (Ambron et al., 1994) that may apply to memory mechanisms is that certain cytoplasmic proteins that can regulate gene transcription are imported into the nucleus after second messengers released during either injury 
or learning expose hidden nuclear localization sequences in the proteins (Walters et al., 1994).

\section{References}

Alberini CM, Ghirardi M, Metz R, Kandel ER (1994) C/EBP is an immediate-early gene required for the consolidation of long-term facilitation in Aplysia. Cell 76:1099-1114.

Aldskogius H, Arvidsson J, Grant G (1992) Axotomy-induced changes in primary sensory neurons. In: Sensory neurons: diversity, development, and plasticity (Scott SA, ed), pp 363-383. New York: Oxford UP.

Ambron RT, Schmied R, Huang CC, Smedman M (1992) A signal sequence mediates the retrograde transport of proteins from the axon periphery to the cell body and then into the nucleus. J Neurosci 12: 2813-2818.

Ambron RT, Zhang X-P, Povelones MT (1994) Axoplasm extruded from injured nerves increases life expectancy and growth when injected into Aplysia neurons. Soc Neurosci Abstr, in press.

Bacskai BJ, Hochner B, Mahaut-Smith M, Adams SR, Kaang BK, Kandel ER, Tsien RY (1993) Spatially resolved dynamics of cAMP and protein kinase A subunits in Aplysia sensory neurons. Science 260: 222-226.

Bailey CH, Chen M (1988) Long-term memory in Aplysia modulates the total number of varicosities of single identified sensory neurons. Proc Natl Acad Sci USA 85:2373-2377.

Billy AJ, Walters ET (1987) Receptive field plasticity and somatotopic organization of pleural mechanosensory/nociceptive neurons of Aplysia. Soc Neurosci Abstr 13:1393.

Billy AJ, Walters ET (1989) Long-term expansion and sensitization of mechanosensory receptive fields in Aplysia support an activitydependent model of whole-cell sensory plasticity. J Neurosci 9:12541262.

Bulloch AGM (1985) Development and plasticity of the molluscan nervous system. In: The mollusca, Vol 8, Neurobiology and behavior, Pt 1 (Willows AOD, ed), pp 335-409. New York: Academic.

Byrne JH (1987) Cellular analysis of associative lcarning. Physiol Rev 67:329-439.

Carew TJ, Sahley CL (1986) Invertebrate learning and memory: from behavior to molecules. Annu Rev Neurosci 9:435-487.

Clark GA, Kandel ER (1993) Induction of long-term facilitation in Aplysia sensory neurons by local application of serotonin to remote synapses. Proc Natl Acad Sci USA 90:11411-11315.

Clatworthy AL, Walters ET (1993) Rapid amplification and facilitation of mechanosensory discharge in Aplysia by noxious stimulation. J Neurophysiol 70:1181-1194.

Clatworthy AL, Walters ET (1994) Comparative analysis of hyperexcitability and synaptic facilitation induced by nerve injury in two populations of mechanosensory neurones of Aplysia californica. J Exp Biol 190:217-238

Clatworthy AL, Castro GA, Budelmann BU, Walters ET (1994) Induction of a cellular defense reaction is accompanied by an increase in sensory neuron excitability in Aplysia. J Neurosci 14:3263-3270.

Cragg BG (1970) What is the signal for chromatolysis? Brain Res 23: $1-21$.

Dale N, Kandel ER, Schacher S (1987) Serotonin produces long-term changes in the excitability of Aplysia sensory neurons in culture that depend on new protein synthesis. J Neurosci 7:2232-2238.

Dash PK, Hochner B, Kandel ER (1990) Injection of the cAMPresponsive element into the nucleus of Aplysia sensory neurons blocks long-term facilitation. Nature 345:718-721.

Dulin MF, Walters ET (1993) Similar alterations of sensory and motor neurons in Aplysia persist after regeneration. Soc Neurosci Abstr 19: 578.

Dulin MF, Billy AJ, Steffensen I, Waltcrs ET (1994) Somatotopic organization and injury-induced plasticity in pleural sensory neurons of Aplysia. Soc Neurosci Abstr, in press.

Eskin A, Garcia KS, Byrne JH (1989) Information storage in the nervous system of Aplysia: specific proteins affected by serotonin and cAMP. Proc Natl Acad Sci USA 86:2458-2462.

Fawcett JW, Keynes RJ (1990) Peripheral nerve regeneration. Annu Rev Neurosci 13:43-60.

Fredman SM (1987) Intracellular staining of neurons with nickellysine. J Neurosci Methods 20:181-194.

Glanzman DL, Kandel ER, Schacher S (1989) Identified target motor neuron regulates neurite outgrowth and synapse formation of Aplysia sensory neurons in vitro. Neuron 3:441-450.

Gunstream JD, Castro GA, Walters ET (1994) Injury-induced plasticity of Aplysia sensory neurons depends on retrograde axonal transport, gene transcription, and protein synthesis. Soc Neurosci Abstr 20: in press.

Haas CA, Donath C, Kreutzberg GW (1993) Differential expression of immediate early genes after transection of the facial nerve. Neuroscience 53:91-99.

Hawkins RD, Abrams TW, Carew TJ, Kandel ER (1983) A cellular mechanism of classical conditioning in Aplysia: activity-dependent amplification of presynaptic facilitation. Science 219:400-404.

Hawkins RD, Kandel ER, Siegelbaum SA (1993) Learning to modulate transmitter release: themes and variations in synaptic plasticity. Annu Rev Neurosci 16:625-665.

Herdegen T, Fiallos-Estrada CE, Schmid W, Bravo R, Zimmermann $M$ (1992) The transcription factors c-JUN, JUN D and CREB, but not FOS and KROX-24, are differentially regulated in axotomized neurons following transection of rat sciatic nerve. Brain Res Mol Brain Res 14:155-165.

Kajander KC, and Bennett GJ (1992) Onset of a painful peripheral neuropathy in rat: a partial and differential deafferentation and spontaneous discharge in A beta and A delta primary afferent neurons. $J$ Neurophysiol 68:734-744.

Kandel ER (1979) Behavioral biology of Aplysia. San Francisco: Freeman.

Kasai H, Petersen O (1994) Spatial dynamics of second messengers: $\mathrm{IP}_{3}$ and cAMP as long-range and associative messengers. Trends Neurosci 3:95-101.

Klein M, Hochner B, Kandel ER (1986) Facilitatory transmitters and cAMP can modulate accommodation as well as transmitter release in Aplysia sensory neurons: evidence for parallel processing in a single cell. Proc Natl Acad Sci USA 83:7994-7998.

Kocher L, Anton F, Reeh PW, Handwerker HO (1987) The effect of carrageenan-induced inflammation on the sensitivity of unmyelinated skin nociceptors in the rat. Pain 29:363-373.

Koike H, Matsumoto H, Umitsu Y (1989) Selective axonal transport in a single cholinergic axon of Aplysia - role of colchicine-resistant microtubules. Neuroscience 32:539-555.

Konorski J (1948) Conditioned reflexes and neuron organization. Cambridge: Cambridge UP.

Lieberman AR (1971) The axon reaction: a review of the principal features of the perikaryal response to axon injury. Int Rev Neurobiol 14:49-124.

Lu X, Richardson PM (1991) Inflammation near the nerve cell body enhances axonal regeneration. $J$ Neurosci 11:972-978.

Mackey SL, Kandel ER, Hawkins RD (1989) Identified serotonergic neurons LCB1 and RCB1 in the cerebral ganglia of Aplysia produce presynaptic facilitation of siphon sensory neurons. J Neurosci 9:42274235.

Mayford M, Barzilai A, Keller F, Schacher S, Kandel ER (1992) Modulation of an NCAM-related adhesion molecule with long-term synaptic plasticity in Aplysia. Science 256:638-644.

Montarolo PG, Goelet P, Castellucci VF, Morgan J, Kandel ER, Schacher $S$ (1986) A critical period for macromolecular synthesis in longterm heterosynaptic facilitation in Aplysia. Science 234:1249-1254.

Nazif FA, Byrne JH, Cleary LJ (1991) cAMP induces long-term morphological changes in sensory neurons of Aplysia. Brain Res 539:324327.

Noel F, Scholz KP, Eskin A, Byrne JH (1991) Common set of proteins in Aplysia sensory neurons affected by an in vitro analogue of longterm sensitization training, 5-HT and cAMP. Brain Res 568:67-75.

Schacher, S, Proshansky E (1983) Neurite regeneration by Aplysia neurons in dissociated cell culture: modulation by Aplysia hemolymph and the presence of the initial axonal segment. J Neurosci 12 : 2403-2413.

Schmied R, Huang C-C, Zhang X-P, Ambron DA, Ambron RT (1993) Endogenous axoplasmic proteins and proteins containing nuclear localization signal sequences use the retrograde axonal transport/nuclear import pathway in Aplysia neurons. J Neurosci 13:4064-4071.

Scholz KP, Byrne JH (1987) Long-term sensitization in Aplysia: biophysical correlates in tail sensory neurons. Science 235:685-687.

Singer PA, Mehler S, Fernandez HL (1982) Blockade of retrograde axonal transport delays the onset of metabolic and morphologic changes induced by axotomy. J Neurosci 2:1299-1306. 
Soiefer AI, Moretto A, Spencer PS, Sabri MI (1988) Axotomy-induced ornithine decarboxylase activity in the mouse dorsal root ganglion is inhibited by the vinca alkaloids. Neurochem Res 13:1169-1173.

Titmus MJ, Faber DS (1990) Axotomy-induced alterations in the electrophysiological characteristics of neurons. Prog Neurobiol 35:151.

Wall PD, Gutnick M (1974) Properties of afferent nerve impulses originating from a neuroma. Nature 248:740-743.

Walters ET (1987a) Site-specific sensitization of defensive reflexes in Aplysia: a simple model of long-term hyperalgesia. J Neurosci 7:400407.

Walters ET (1987b) Multiple sensory neuronal correlates of site-specific sensitization in Aplysia. J Neurosci 7:408-417.

Walters ET (1994) Injury-related behavior and neuronal plasticity: an evolutionary perspective on sensitization, hyperalgesia and analgesia Int Rev Neurobiol 36: in press.

Walters ET, Byrne JH, Carew TJ, Kandel ER (1983a) Mechanoafferent neurons innervating tail of Aplysia. I. Response properties and synaptic connections. J Neurophysiol 50:1522-1542.

Walters ET, Byme JH, Carew TJ, Kandel ER (1983b) Mechanoafferent neurons innervating tail of Aplysia. II. Modulation by sensitizing stimulation. J Neurophysiol 50:1543-1559.

Walters ET, Alizadeh H, Castro GA (1991) Similar neuronal alterations induced by axonal injury and learning in Aplysia. Science 253: 797-799.

Walters ET, Dulin, MF, Zhang, X-P, Ambron, RT (1994) Plasticity induced by injecting Aplysia neurons with molecules transported to the soma after axonal crush suggests a conserved mechanism for injury reactions and memory. Soc Neurosci Abstr, in press.

Watson WE (1968) Observations on the nucleolar and total body nucleic acid of injured nerve cells. J Physiol (Lond) 196:655-676.

Wu W, Mathew TC, Miller FD (1993) Evidence that the loss of homeostatic signals induces regeneration-associated alterations in neuronal gene expression. Dev Biol 158:456-466. 